\section{EFFECT OF PARTIALLY HYDROLYZED AND EXTENSIVELY HYDROLYZED MILK FORMULAS ON THE ORAL TOLERANCE DEVELOPMENT IN INFANTS AT RISK OF ALLERGY}

\author{
C. Dupont ${ }^{1}$, R. Amar ${ }^{2}$, J.-P. Basuyau ${ }^{2}$, \\ P. Soulaines ${ }^{1}$, M.-F. Grancher ${ }^{2}$, R. Fritsché ${ }^{3}$, \\ S. Pecquet ${ }^{4}$, E. Mallet ${ }^{2}$ \\ ${ }^{1}$ Université Paris-Descartes, Hôpital Saint Vincent \\ de Paul, Paris, ${ }^{2}$ Hôpital Charles Nicolle, CIC \\ INSERM 204, Rouen, France, ${ }^{3}$ Nestle Research \\ Center, Lausanne, ${ }^{4}$ Nestlé Nutrition, \\ Vevey, Switzerland
}

Objective: A randomized, double-blind controlled study compared breastfeeding, partially- and extensively-hydrolyzed protein formulas ( $\mathrm{pHF}$ and eHF) in their effect on cow' milk allergy (CMA) and atopic symptoms in infants at risk of allergy.

Methods: Healthy, full-term infants were randomized to receive a whey pHF or a whey eHF, either exclusively from birth to 4 months of age or at weaning when mothers were willing to breastfeed child. The population consisted of 141 (ITT) and 104 (PP) children in the pHF group and 138 (ITT) and 90 (PP) in the eHF group, of whom respectively 40 (ITT) and 32 (PP) and 38 (ITT) and 31 (PP) were partially breastfed. At 4 months, a milk oral food challenge was performed and at 4 and 12 months, skin prick tests with various food antigens and plasma immunoglobulin concentrations were measured. Standard growth parameters were followed.

Results: The incidences of CMA and of reactions to other food antigens were similarly low in all groups. Total IgE plasma concentrations increased between 4 and 12 months in all groups but increased significantly $(p=0.048)$ less with $\mathrm{pHF}(16.92 \pm 47.11$ $\mathrm{kU} / \mathrm{L})$ than with eHF $(23.68 \pm 41.29 \mathrm{kU} / \mathrm{L})$. There was an unexpectedly higher hemoglobin level in infants fed a pHF or an eHF compared to breastfeeding. Infants in all groups grew normally according to WHO standards.

Conclusion: The whey pHF and the eHF reduced the risk of CMA and of atopic symptoms similarly. The $\mathrm{pHF}$ was superior in reducing IgE levels during the first year of life.

\section{STUDY TO INVESTIGATE POTENTIAL BENEFITS OF PROBIOTICS IN YOGURT IN CHILDREN ATTENDING SCHOOL, A PATIENT- ORIENTED, DOUBLE-BLIND, CLUSTER- RANDOMIZED, PLACEBO-CONTROLLED, CLINICAL TRIAL}

T.P. Tan'1, D.J. Merenstein ${ }^{1}$, J. Gonzalez', A. Guimarães Young ${ }^{1}$, R.F. Roberts², M.E. Sanders ${ }^{3}$, S. Petterson ${ }^{4}$

${ }^{1}$ Department of Family Medicine, Georgetown University Medical Center, Washington, DC, ${ }^{2}$ Department of Food Science, The Pennsylvania State University, University Park, PA, ${ }^{3}$ Dairy \&

Food Culture Technologies, Centennial, CO,

${ }^{4}$ Robert Graham Center, Washington, DC, USA

Background and aims: Functional foods, especially yogurt, are attractive delivery agents for probiotics due to their popularity with parents and children; however, few commercially available products have strong clinical-based evidence. Our primary objective was to determine if consumption of a probiotic-supplemented yogurt-based beverage containing Bifidobacterium animalis ssp. Lactis (B. lactis) BB-12, at a high dose, reduces daycare absences in children ages 2-4.

Methods: We conducted a double-blinded, randomized, placebo-controlled, allocation concealment clinical trial with 188 healthy children ages 2-4 years attending daycare at least 3 days per week. Participants consumed 4 ounces of active or control drink for 90 consecutive days. Active intervention was a yogurt-based drink supplemented with BB-12. Placebo contained the two cultures commonly found in all yogurts without BB-12 and was indistinguishable from the active beverage.

Results: There were no significant differences in days of missed daycare due to illness between the groups, active (2.54 days absent/100 school days) and control (2.42 days absent/100 school days). A subset of less healthy children at baseline $(\mathrm{N}=59)$ showed a significant difference in rates of constipation between the groups, active (2.76 days affected/100 days) and control (0.68 days affected/100 days).

Conclusions: Consumption of a BB-12 drink did not reduce daycare absences due to illness in healthy children; however, the yogurt was found to be safe and well-tolerated. As there are many probiotic 